\title{
The Cauchy Problem for Darboux Integrable Systems and Non-Linear d'Alembert Formulas ${ }^{\star}$
}

\author{
Ian M. ANDERSON and Mark E. FELS
}

Utah State University, Logan Utah, USA

E-mail:Ian.Anderson@usu.edu,Mark.Fels@usu.edu

Received October 08, 2012, in final form February 20, 2013; Published online February 27, 2013 http://dx.doi.org/10.3842/SIGMA.2013.017

\begin{abstract}
To every Darboux integrable system there is an associated Lie group $G$ which is a fundamental invariant of the system and which we call the Vessiot group. This article shows that solving the Cauchy problem for a Darboux integrable partial differential equation can be reduced to solving an equation of Lie type for the Vessiot group $G$. If the Vessiot group $G$ is solvable then the Cauchy problem can be solved by quadratures. This allows us to give explicit integral formulas, similar to the well known d'Alembert's formula for the wave equation, to the initial value problem with generic non-characteristic initial data.
\end{abstract}

Key words: Cauchy problem; Darboux integrability; exterior differential systems; d'Alembert's formula

2010 Mathematics Subject Classification: 58A15; 35L52; 58J70; 35A30; 34A26

Dedicated to our good friend and collaborator Peter Olver on the occasion of his $60^{\text {th }}$ birthday.

\section{Introduction}

The solution to the classical wave equation $u_{t t}-u_{x x}=0$ with initial data $u(0, x)=a(x)$ and $u_{t}(0, x)=b(x)$ is given by the well-known d'Alembert's formula

$$
u(t, x)=\frac{1}{2}(a(x-t)+a(x+t))+\frac{1}{2} \int_{x-t}^{x+t} b(\xi) d \xi .
$$

In this article we characterize a broad class of differential equations where the solution to the Cauchy problem can be expressed in terms of the initial data by quadratures as in (1.1).

The family of equations which we identify that can be solved in this manner are a subset of the partial differential equations known as Darboux integrable equations. The results we present here are for the classical case of a scalar Darboux integrable equation in the plane but we also illustrate how these results do hold in the more general case of a Darboux integrable exterior differential system. An example of such an equation is the non-linear hyperbolic PDE in the plane

$$
u_{x y}=\frac{u_{x} u_{y}}{u-x} .
$$

With initial data given along $y=x$ by $u(x, x)=f(x)$ and $u_{x}(x, x)=g(x)$ we find that the analogue to (1.1) (in null coordinates) is

$$
u=x+(f(y)-y) e^{\int_{x}^{y} G(t) d t}+e^{-\int_{0}^{x} G(t) d t}\left(\int_{x}^{y} e^{\int_{0}^{s} G(t) d t} d s\right),
$$

\footnotetext{
* This paper is a contribution to the Special Issue "Symmetries of Differential Equations: Frames, Invariants and Applications". The full collection is available at http://www.emis.de/journals/SIGMA/SDE2012.html
} 
where

$$
G(t)=\frac{g(t)}{t-f(t)} .
$$

A fundamental invariant of a Darboux integrable system, called the Vessiot group, was introduced in [4]. The Vessiot group $G$ is a Lie group which plays an essential role in the analysis of many of the geometric properties of Darboux integrable equations [1,3]. This is also true when solving the Cauchy problem for these systems as can be seen by the following theorem.

Theorem 1.1. Let $F\left(x, y, u, u_{x}, u_{y}, u_{x x}, u_{x y}, u_{y y}\right)=0$ be a hyperbolic PDE in the plane. If $F$ (or its prolongation) is Darboux integrable then the initial value problem can be solved by integrating an equation of fundamental Lie type for the Vessiot group $G$. If $G$ is simply connected and solvable, then the initial value problem can be solved by quadratures.

Theorem 1.1 can be viewed as a generalization of the classical theorem of Sophus Lie on solving an ordinary differential equation by quadratures [15]. This theorem states that the general solution, or the solution to the initial value problem, of an $n^{\text {th }}$ order ordinary differential equation with an $n$-dimensional solvable symmetry group can be solved by quadratures. While this classical result on integrating ODE's has motivated our work, there is one fundamental difference between this classical result for ODE and Theorem 1.1. In Theorem 1.1 the group $G$ is not a symmetry group of the $\mathrm{PDE} F=0$.

In [8] it is shown how the initial value problem for Darboux integrable hyperbolic systems can be solved using the Frobenius theorem. The approach we take here is quite different. By using the quotient representation for Darboux integrable hyperbolic Pfaffians systems constructed in [4], we show that the initial value problem can be solved by solving an equation of fundamental Lie type for the Vessiot group $G$. This, in turn, allows us to conclude that if the group $G$ is solvable, then the initial value problem can be solved by quadratures. We illustrate this with a number of examples. The relationship between our approach and the approach given in [8] is described in Appendix A. An example given in the appendix compares the two approaches.

\section{Pfaffian systems and reduction}

In this section we give the definition of a Pfaffian system and summarize some basic facts about their reduction by a symmetry group.

\subsection{Pfaffian systems}

A constant rank Pfaffian system is given by a constant rank sub-bundle $I \subset T^{*} M$. An integral manifold of $I$ is a smooth immersion $s: N \rightarrow M$ such that $s^{*} I=0$. If $N$ is an open interval, then we call $s$ an integral curve of $I,[6]$.

A local first integral of a Pfaffian system $I$ is a smooth function $F: U \rightarrow \mathbb{R}$, defined on an open set $U \subset M$, such that $d F \in I$. For each point $x \in M$ we define

$$
I_{x}^{\infty}=\left\{d F_{x} \mid F \text { is a local first integral, defined about } x\right\} .
$$

We shall assume that $I^{\infty}=\bigcup_{x \in M} I_{x}^{\infty}$ is a constant rank bundle on $M$. It is easy to verify that $I^{\infty}$ is the (unique) maximal, completely integrable, Pfaffian sub-system of $I$. Granted additional regularity conditions (see below), the bundle $I^{\infty}$ can be computed algorithmically using the derived sequence of $I$.

The derived system $I^{\prime} \subset I$ of a Pfaffian system $I$ is defined pointwise by

$$
I_{x}^{\prime}=\operatorname{span}\left\{\theta_{x} \mid \theta \in \mathcal{S}(I) \text { and } d \theta \equiv 0 \bmod I\right\},
$$


where $\mathcal{S}(I)$ are the sections of $I$. The system $I$ is integrable if it satisfies the Frobenius condition $I^{\prime}=I$. Letting $I^{(0)}=I$, and assuming $I^{(k)}$ is constant rank, we define the derived sequence inductively by

$$
I^{(k+1)}=\left(I^{(k)}\right)^{\prime}, \quad k=0,1, \ldots, N
$$

where $N$ is the smallest integer such that $I^{(N+1)}=I^{(N)}$. Therefore $I^{\infty}=I^{(N)}$ whenever the sets $I^{(k)}$ are constant rank bundles. More information about the derived sequence can be found in $[6]$.

\subsection{Reduction of Pfaffian systems}

A Lie group $G$ acting on $M$ is a symmetry group of the Pfaffian system $I$ if

$$
g^{*} I=I, \quad \text { for all } g \in G \text {. }
$$

The group $G$ acts regularly on $M$ if the quotient map $\mathbf{q}_{G}: M \rightarrow M / G$ is a smooth submersion. Let $\Gamma$ denote the Lie algebra of infinitesimal generators for the action of $G$ on $M$ and let $\Gamma \subset T M$ be its pointwise span. Then $\boldsymbol{\Gamma}=\operatorname{ker}\left(\mathbf{q}_{G *}\right)$. Assume from now on that the action of $G$ on $M$ is regular and a symmetry group of $I$.

The reduction $I / G$ of $I$ by the symmetry group $G$ is defined by

$$
I / G=\left\{\bar{\theta} \in \Lambda^{1}(M / G) \mid \mathbf{q}_{G}^{*} \bar{\theta} \in I\right\} .
$$

Let $\operatorname{ann}(I) \subset T M$ be the annihilating space of $I$,

$$
\operatorname{ann}(I)=\{X \in T M \mid \theta(X)=0 \text { for all } \theta \in I\} .
$$

It is easy to check that $I / G$ is constant rank if and only if $\boldsymbol{\Gamma} \cap \operatorname{ann}(I)$ is constant rank [2] in which case

$$
\operatorname{rank}(I / G)=\operatorname{rank}(I)-(\operatorname{rank}(\boldsymbol{\Gamma})-\operatorname{rank}(\boldsymbol{\Gamma} \cap \operatorname{ann}(I)))=\operatorname{rank}(\operatorname{ann}(\boldsymbol{\Gamma}) \cap I) .
$$

The annihilating space $\operatorname{ann}(I)$ is $G$ invariant and satisfies [12],

$$
\mathbf{q}_{G *}(\operatorname{ann}(I))=\operatorname{ann}(I / G) .
$$

For examples on computing $I / G$, applications, and more information about its properties see $[1$, 2, 3, 12].

The symmetry group $G$ is said to act transversally to the Pfaffian system $I$ if

$$
\operatorname{ker}\left(\mathbf{q}_{G *}\right) \cap \operatorname{ann}(I)=0 .
$$

The following basic theorem, which follows directly from Theorem 2.2 in [1], illustrates the importance of the transversality condition (2.3).

Theorem 2.1. Let $G$ be a symmetry group of the constant rank Pfaffian system $I$ on $M$ acting freely and regularly on $M$ and transversally to $I^{\prime}$. Then $I / G$ is a constant rank Pfaffian system and $\left(I^{\prime}\right) / G=(I / G)^{\prime}$. Furthermore, if $\gamma:(a, b) \rightarrow M / G$ is a one-dimensional integral manifold of $I / G$, then through each point $p \in M$ satisfying $\mathbf{q}_{G}(p)=\gamma\left(t_{0}\right)$ there exists a unique lift $\sigma:(a, b) \rightarrow M$ of $\gamma$ which is an integral manifold of I satisfying $\sigma\left(t_{0}\right)=p$. The lifted curve $\sigma$ can be found by solving an equation of fundamental Lie type. 
Let $\mathfrak{g}$ be the Lie algebra of $G$. Then, given a curve $\alpha:(a, b) \rightarrow \mathfrak{g}$, the system of ODE

$$
\dot{\lambda}(t)=\left(L_{\lambda}\right)_{*}(\alpha(t))
$$

for the curve $\lambda: \mathbb{R} \rightarrow G$ is called an equation of fundamental Lie type. Given $\alpha$ in equation (2.4), the curve $\lambda$ can be found globally over the entire interval $(a, b)$. See [5, p. 55] for more information about equations of Lie type. If $G$ is simply connected and $\mathfrak{g}$ is solvable, then an equation of fundamental Lie type can be solved by quadratures, see [5, Proposition 4, p. 60].

Proof. The first statement in Theorem 2.1 is proved in [2]. For the second statement concerning the lifting of integral curves $\gamma$ we begin by noting that since $G$ is free we may, by using a $G$ invariant Riemannian metric on $M$, write

$$
I=\mathbf{q}_{G}^{*}(I / G) \oplus K,
$$

where $\mathbf{q}^{*}(I / G)$ is the pullback bundle and $K$ is its $G$-invariant orthogonal complement in $I$. The transversality condition (2.3) implies that

$$
\operatorname{rank}(K)=\operatorname{dim} G \quad \text { and } \quad \operatorname{ann}(K) \cap \operatorname{ker}\left(\mathbf{q}_{G *}\right)=0 .
$$

Therefore $\operatorname{ann}(K)$ is a horizontal space for the principal bundle $M \rightarrow M / G$. Consequently, since $\gamma$ is an integral curve of $I / G$, a lift $\sigma$ which is an integral curve of $I$ satisfies on account of equation (2.5), $\sigma^{*}(K)=0$. Therefore $\sigma$ is a horizontal lift for the connection $\operatorname{ann}(K)$ on the principal bundle $M$. But the system of ODE determining a horizontal lift for a connection on a principal bundle is precisely an equation of fundamental Lie type for the structure group $G$, see $[14$, p. 69].

Remark 2.2. There are two standard ways to find a lift $\sigma:(a, b) \rightarrow M$ of $\gamma$. To describe them let $p \in M$ satisfy $\mathbf{q}_{G}(p)=\gamma\left(t_{0}\right)$. We now describe how to find the lift satisfying $\sigma\left(t_{0}\right)=p$.

The first way is to start with any lift $\hat{\sigma}:(a, b) \rightarrow M$ of $\gamma$ satisfying $\hat{\sigma}\left(t_{0}\right)=p$. Then define a lift $\sigma(t)=\lambda(t) \hat{\sigma}(t)$, where $\lambda:(a, b) \rightarrow G$ and $\lambda\left(t_{0}\right)=e_{G}$. The requirement that $\sigma$ be an integral curve of $I$ is equivalent to $\lambda$ satisfying an equation of fundamental Lie type. The equation of Lie type is easily determined algebraically from $I$ and $\hat{\sigma}$.

The second way to find the lift $\sigma$ is to assume $\gamma:(a, b) \rightarrow M / G$ is an embedding. Then let $S=\gamma(a, b)$ and $P=\mathbf{q}_{G}^{-1}(S)$. Theorem 2.1 states that the restriction $(I)_{P}$ is the Pfaffian system for an equation of fundamental Lie type in the sense of [10] or [11]. We then compute the maximal integral manifold through $p \in P$ in order to find $\gamma$.

\section{$3 \quad$ Hyperbolic Pfaffian systems}

A constant rank Pfaffian system $I$ is said to be hyperbolic of class $s$ (see [8]) if the following holds. About each point $x \in M$ there exists an open set $U$ and a coframe $\left\{\theta^{1}, \ldots, \theta^{s}, \widehat{\omega}, \widehat{\pi}, \breve{\omega}, \check{\pi}\right\}$ on $U$ such that

$$
I=\operatorname{span}\left\{\theta^{1}, \ldots, \theta^{s}\right\}
$$

and the following structure equations hold,

$$
\begin{aligned}
& d \theta^{i} \equiv 0 \quad \bmod I, \quad 1 \leqslant i \leqslant s-2, \\
& d \theta^{s-1} \equiv \widehat{\omega} \wedge \widehat{\pi} \quad \bmod I, \\
& d \theta^{s} \equiv \breve{\omega} \wedge \check{\pi} \quad \bmod I .
\end{aligned}
$$


The two Pfaffian systems $\widehat{V}$ and $\breve{V}$ defined by

$$
\widehat{V}=\operatorname{span}\left\{\theta^{i}, \widehat{\pi}, \widehat{\omega}\right\}, \quad \check{V}=\operatorname{span}\left\{\theta^{i}, \check{\pi}, \breve{\omega}\right\}
$$

are called the associated characteristic or singular systems of $I$. They are important invariants of $I$. Let $\widehat{V}^{\infty}$ and $\breve{V}^{\infty}$ be the corresponding subspaces of first integrals for the singular Pfaffian systems $\widehat{V}$ and $\breve{V}$ in (3.1) of the hyperbolic Pfaffian system $I$. A locally defined function $F$ satisfying $d F \in \widehat{V}^{\infty}$ or $d F \in \breve{V}^{\infty}$ is called an intermediate integral of $I$ or a Darboux invariant of $I$. We will call $\widehat{V}^{\infty}$ and $\breve{V}^{\infty}$ the spaces of intermediate integrals of $I$. The hyperbolic system $I$ is said to be Darboux integrable if $I^{\infty}=0$, and

$$
\widehat{V}+\breve{V}^{\infty}=T^{*} M \quad \text { and } \quad \widehat{V}^{\infty}+\check{V}=T^{*} M .
$$

See [4] and Theorem 4.3 in [1]. In particular Theorem 4.3 in [1] shows that the conditions in (3.2) imply

$$
\widehat{V}^{\infty} \cap \check{V}^{\infty}=0 .
$$

For more information about hyperbolic Pfaffian systems see the article [8] where the general theory of hyperbolic exterior differential systems is developed in detail. The definition of a hyperbolic exterior differential system is generalized in [4], see Section 5.2.

The characteristic directions for a hyperbolic system $I$ with singular systems $\widehat{V}$ and $\breve{V}$ are $\operatorname{ann}(\widehat{V})$ and $\operatorname{ann}(\breve{V})[8]$. A non-characteristic integral curve is an immersion $\gamma:(a, b) \rightarrow M$ which is a one-dimensional integral manifold of $I$ such that

$$
\dot{\gamma}(t) \notin \operatorname{ann}(\widehat{V}) \quad \text { and } \quad \dot{\gamma}(t) \notin \operatorname{ann}(\breve{V}), \quad \text { for all } t \in(a, b) \text {. }
$$

Given a non-characteristic integral curve $\gamma:(a, b) \rightarrow M$ of $I$, a solution to the Cauchy or initial value problem for $\gamma$ is a 2-dimensional integral manifold $s: N \rightarrow M$ of $I$ such that $\gamma(a, b) \subset s(N)$. A local solution to the Cauchy problem about a point $x \in \gamma(a, b)$ is an open neighbourhood $U \subset M, x \in U$, and a 2-dimensional integral manifold $s: N \rightarrow U$ of $I$ such that $\gamma(a, b) \cap U \subset s(N)$.

Hyperbolic Pfaffian systems of class $s=3$ are closely related to hyperbolic partial differential equations in the plane [13]. Specifically, a hyperbolic PDE in the plane

$$
F\left(x, y, u, u_{x}, u_{y}, u_{x x}, u_{x y}, u_{y y}\right)=0
$$

defines a 7-dimensional submanifold $M \subset J^{2}\left(\mathbb{R}^{2}, \mathbb{R}\right)$. Let $I$ be the rank 3 Pfaffian system which is the restriction of the rank 3 contact system on $J^{2}\left(\mathbb{R}^{2}, \mathbb{R}\right)$ to $M$. The following structure equations proven in [13] show that $I$ is a class $s=3$ hyperbolic Pfaffian system.

Theorem 3.1. Let $I$ be the rank 3 Pfaffian system on the 7-manifold $M$ defined by the hyperbolic PDE in the plane in (3.4). About each point $p \in M$ there exists a local coframe $\left\{\theta^{i}, \omega^{a}, \pi^{a}\right\}_{0 \leqslant i \leqslant 2,1 \leqslant a \leqslant 2}$ such that

$$
I=\operatorname{span}\left\{\theta^{0}, \theta^{1}, \theta^{2}\right\},
$$

and

$$
\begin{aligned}
& d \theta^{0}=\theta^{1} \wedge \omega^{1}+\theta^{2} \wedge \omega^{2} \quad \bmod \left\{\theta^{0}\right\}, \\
& d \theta^{1}=\omega^{1} \wedge \pi^{1}+\mu_{1} \theta^{2} \wedge \pi^{2} \quad \bmod \left\{\theta^{0}, \theta^{1}\right\}, \\
& d \theta^{2}=\omega^{2} \wedge \pi^{2}+\mu_{2} \theta^{1} \wedge \pi^{1} \quad \bmod \left\{\theta^{0}, \theta^{2}\right\},
\end{aligned}
$$

where $\mu_{1}, \mu_{2}$ are the Monge-Ampere invariants. The invariant conditions $\mu_{1}=\mu_{2}=0$ are satisfied if and only if (3.4) is locally a Monge-Ampere equation.

The article by Gardner and Kamran [13] gives an algorithm to construct the coframe in equations (3.5). The corresponding singular systems $\widehat{V}$ and $\breve{V}$ are denoted by $C\left(\mathcal{I}_{F}, d M_{1}\right)$ and $C\left(\mathcal{I}_{F}, d M_{2}\right)$ in [13], and in frame (3.5), $\widehat{V}=\left\{\theta^{0}, \theta^{1}, \theta^{2}, \omega^{1}, \pi^{1}\right\}$ and $\check{V}=\left\{\theta^{0}, \theta^{1}, \theta^{2}, \omega^{2}, \pi^{2}\right\}$. 


\section{The Vessiot group and the quotient representation}

\subsection{The quotient representation}

We now explain the fundamental role played by the quotient of a Pfaffian system by a symmetry group in the theory of Darboux integrability. See [1, Section 6, p. 20] (with $L=G_{\text {diag }}$ ) for more information and detailed proofs of the following key theorem.

Theorem 4.1. Let $K_{i}, i=1,2$ be constant rank Pfaffian systems on $M_{i}, i=1,2$ of codimension 2 , and satisfying $K_{i}^{\infty}=0$. Consider a Lie group $G$ which acts freely and regularly on $M_{i}$, is a common symmetry group of both $K_{1}$ and $K_{2}$ and acts transversely to $K_{1}$ and $K_{2}$. Assume also that the action of the diagonal subgroup $G_{\text {diag }} \subset G \times G$ on $M_{1} \times M_{2}$ is regular and acts transversely to $K_{1}^{\prime}+K_{2}^{\prime}$.

(i) The sum $K_{1}+K_{2}$ on $M_{1} \times M_{2}$ is a constant rank, Darboux integrable, hyperbolic Pfaffian system.

(ii) The singular Pfaffian systems for $K_{1}+K_{2}$ are

$$
\widehat{W}=K_{1}+T^{*} M_{2} \quad \text { and } \quad \widetilde{W}=T^{*} M_{1}+K_{2} .
$$

(iii) The quotient differential system $I=\left(K_{1}+K_{2}\right) / G_{\text {diag }}$ on $M=\left(M_{1} \times M_{2}\right) / G_{\text {diag }}$ is a constant rank hyperbolic Pfaffian system which is Darboux integrable.

(iv) The singular Pfaffian systems for the quotient system I are

$$
\widehat{V}=\left(K_{1}+T^{*} M_{2}\right) / G_{\text {diag }}=\widehat{W} / G_{\text {diag }} \quad \text { and } \quad \breve{V}=\left(T^{*} M_{1}+K_{2}\right) / G_{\text {diag }}=\widetilde{W} / G_{\text {diag }} .
$$

$(v)$ The spaces of intermediate integrals for I are

$$
\widehat{V}^{\infty}=\left(0+T^{*} M_{2}\right) / G_{\text {diag }}=\widehat{W}^{\infty} / G_{\text {diag }} \quad \text { and } \quad \check{V}^{\infty}=\left(T^{*} M_{1}+0\right) / G_{\text {diag }}=\widetilde{W}^{\infty} / G_{\text {diag }} .
$$

The sums of the type $K_{1}+K_{2}$ in Theorem 4.1 are defined precisely as

$$
K_{1}+K_{2}=\pi_{1}^{*}\left(K_{1}\right)+\pi_{2}^{*}\left(K_{2}\right),
$$

where $\pi_{i}: M_{1} \times M_{2} \rightarrow M_{i}$.

Theorem 4.1 shows how Darboux integrable hyperbolic Pfaffian systems can be constructed using the group reduction of pairs of Pfaffian systems. It is a remarkable fact, established in [4], that the converse is true locally, that is, every Darboux integrable hyperbolic system can be realized locally as a non-trivial quotient of a pair of Pfaffian systems with a common symmetry group. The precise formulation of this result is as follows.

Theorem 4.2. Let $I$ be a Darboux integrable hyperbolic Pfaffian system on a manifold $M$ and let $\widehat{V}$ and $\breve{V}$ be the singular Pfaffian systems as in (3.1). Fix a point $x_{0}$ in $M$ and let

(i) $M_{1}$ and $M_{2}$ be the maximal integral manifolds of $\hat{V}^{\infty}$ and $\breve{V}^{\infty}$ through $x_{0}$, and

(ii) $K_{1}$ and $K_{2}$ be the restrictions of $\widehat{V}$ and $\breve{V}$ to $M_{1}$ and $M_{2}$ respectively.

Then there are open sets $U \subset M, U_{1} \subset M_{1}, U_{2} \subset M_{2}$, each containing $x_{0}$, and a local action of a Lie group $G$ on $U_{1}$ and $U_{2}$ which satisfy the hypothesis of Theorem 4.1 and such that

$$
U=\left(U_{1} \times U_{2}\right) / G_{\text {diag }}, \quad I_{U}=\left(\left(K_{1}+K_{2}\right)_{U_{1} \times U_{2}}\right) / G_{\text {diag }},
$$

and properties $(i v)$ and $(v)$ in Theorem 4.1 hold on $U$. 
The group $G$ appearing in Theorems 4.1 and 4.2 is called the Vessiot group of the Darboux integrable system I. We shall refer to (4.2) or (iii) in Theorem 4.1 as the canonical quotient representation for a Darboux integrable hyperbolic Pfaffian system I.

Remark 4.3. It is an algorithmic (but non-trivial) process to find the group $G$ and its action in Theorem 4.2. The Lie algebra of infinitesimal generators of $G$ can be found algebraically, while finding the action of $G$ may require solving a system of Lie type $[9,10]$.

\section{Solving the Cauchy initial value problem for Darboux integrable systems}

In this section we solve the initial value problem for a Darboux integrable hyperbolic Pfaffian system and give a proof of Theorem 1.1. In the second subsection we outline how to extend these results to the general case of Darboux integrable systems which are not necessarily hyperbolic.

\subsection{The Cauchy problem for hyperbolic systems}

We begin with a key lemma which shows that the lift of a non-characteristic integral curve is again a non-characteristic integral curve.

Lemma 5.1. Let I be a hyperbolic Pfaffian system which is Darboux integrable and let $\left(K_{1}+\right.$ $\left.K_{2}\right) / G_{\text {diag }}$ be the canonical quotient representation of $I$ as in Theorem 4.2 . Let $\gamma:(a, b) \rightarrow M$ be a non-characteristic integral curve of $I$ and let $\sigma:(a, b) \rightarrow M_{1} \times M_{2}$ be a lift of $\gamma$ to a one-dimensional integral curve of $K_{1}+K_{2}$. Then $\sigma$ is a non-characteristic integral curve for $K_{1}+K_{2}$.

The existence of the lifted integral curve $\sigma$ in Lemma 5.1 is guaranteed by Theorem 2.1.

Proof. In parts $(i)$ and $(i i)$ of Theorem 4.1 it is noted that $K_{1}+K_{2}$ is Darboux integrable with characteristic systems $\widehat{W}=\left(K_{1}+T^{*} M_{2}\right)$ and $\widetilde{W}=T^{*} M_{1}+K_{2}$. Therefore, in view of equation (3.3), we prove Lemma 5.1 by showing

$$
\dot{\sigma}(t) \notin \operatorname{ann}(\widehat{W}) \quad \text { and } \quad \dot{\sigma}(t) \notin \operatorname{ann}(\widetilde{W}), \quad \text { for all } t \in(a, b) .
$$

From part (iv) of Theorem 4.1 and equation (2.2) we find that

$$
\begin{aligned}
& \mathbf{q}_{G_{\text {diag }}(\operatorname{ann}(\widehat{W}))}=\operatorname{ann}\left(\widehat{W} / G_{\text {diag }}\right)=\operatorname{ann}(\widehat{V}) \quad \text { and } \\
& \mathbf{q}_{G_{\text {diag }} *}(\operatorname{ann}(\widetilde{W}))=\operatorname{ann}\left(\widetilde{W} / G_{\text {diag }}\right)=\operatorname{ann}(\breve{V}) .
\end{aligned}
$$

Now suppose that $\sigma$ is characteristic at some point $t_{0}$ so that, for instance, $\dot{\sigma}\left(t_{0}\right) \in \operatorname{ann}(\widehat{W})$. Then by (5.1)

$$
\mathbf{q}_{G_{\text {diag }} *}\left(\dot{\sigma}\left(t_{0}\right)\right) \in \operatorname{ann}(\widehat{V})
$$

and hence, since $\sigma$ is a lift of $\gamma, \dot{\gamma}\left(t_{0}\right)=\mathbf{q}_{G_{\text {diag }} *}\left(\dot{\sigma}\left(t_{0}\right)\right) \in \operatorname{ann}(\hat{V})$. This contradicts the fact that $\gamma$ is not characteristic. A similar argument applies if we assume $\dot{\sigma}\left(t_{0}\right) \in \widetilde{W}$.

We also need the following lemma.

Lemma 5.2. Let $\sigma:(a, b) \rightarrow M_{1} \times M_{2}$ be a non-characteristic integral curve of $K_{1}+K_{2}$, where $K_{1}$ and $K_{2}$ satisfy the conditions of Theorem 4.1. Then the curves $\sigma_{i}:(a, b) \rightarrow M_{i}$ defined by

$$
\sigma_{i}=\pi_{i} \circ \sigma
$$

are 1-dimensional integral manifolds of $K_{i}$. 
Proof. By applying (4.1) and (5.2) we find

$$
\sigma_{i}^{*}\left(K_{i}\right)=\sigma^{*} \pi_{i}^{*}\left(K_{i}\right) \subset \sigma^{*}\left(K_{1}+K_{2}\right)=0 .
$$

It then remains to be shown that the maps $\sigma_{i}:(a, b) \rightarrow M_{i}$ are immersions.

Suppose $\dot{\sigma}_{1}\left(t_{0}\right)=\left(\pi_{1 *}(\dot{\sigma})\right)\left(t_{0}\right)=0$. Then on the one-hand,

$$
\dot{\sigma}\left(t_{0}\right) \in \operatorname{ker}\left(\pi_{1 *}\right)=0+T M_{2} .
$$

On the other hand, by writing

$$
K_{1}+K_{2}=\left(K_{1}+T^{*} M_{2}\right) \cap\left(T^{*} M_{1}+K_{2}\right)
$$

we also have that

$$
\dot{\sigma}\left(t_{0}\right) \in \operatorname{ann}\left(K_{1}+K_{2}\right)=\operatorname{ann}\left(K_{1}+T^{*} M_{2}\right) \oplus \operatorname{ann}\left(T^{*} M_{1}+K_{2}\right) .
$$

Therefore, since

$$
\operatorname{ann}\left(K_{1}+T^{*} M_{2}\right) \cap\left(0+T M_{2}\right)=0 \quad \text { and } \quad \operatorname{ann}\left(T^{*} M_{1}+K_{2}\right) \subset\left(0+T M_{2}\right) \text {, }
$$

we get from equations (5.3) and (5.4)

$$
\dot{\sigma}\left(t_{0}\right) \in \operatorname{ann}\left(T^{*} M_{1}+K_{2}\right)=\operatorname{ann}(\widetilde{W}),
$$

which contradicts the hypothesis that $\sigma$ is non-characteristic. A similar argument applies to $\sigma_{2}$ and so we conclude that the maps $\sigma_{i}:(a, b) \rightarrow M_{i}$ are immersions.

The solution to the Cauchy initial value problem can now be given.

Theorem 5.3. Let $\gamma:(a, b) \rightarrow M$ be a non-characteristic integral curve for the Darboux integrable hyperbolic Pfaffian system $I$. Let I have canonical quotient representation $\left(K_{1}+\right.$ $\left.K_{2}\right) / G_{\text {diag }}$, let $\sigma:(a, b) \rightarrow M_{1} \times M_{2}$ be a lift of $\gamma$ to an integral curve of $K_{1}+K_{2}$ and let $\sigma_{i}=\pi_{i} \circ \sigma:(a, b) \rightarrow M_{i}$. Then the smooth function $s:(a, b) \times(a, b) \rightarrow M$ defined by

$$
s\left(t_{1}, t_{2}\right)=\mathbf{q}_{G_{\text {diag }}}\left(\sigma_{1}\left(t_{1}\right), \sigma_{2}\left(t_{2}\right)\right)
$$

solves the Cauchy problem for $\gamma$.

Proof. We first show that $s$ is an integral manifold of $I$. Define $\Sigma:(a, b) \times(a, b) \rightarrow M_{1} \times M_{2}$ by

$$
\Sigma\left(t_{1}, t_{2}\right)=\left(\sigma_{1}\left(t_{1}\right), \sigma_{2}\left(t_{2}\right)\right)
$$

Lemma 5.2 implies that $\Sigma$ is a 2-dimensional integral manifold of $K_{1}+K_{2}$. By the definition of quotient in (2.1), $\mathbf{q}_{G_{\text {diag }}}$ maps integral manifolds to (possibly non-immersed) integral manifolds. The condition that $G_{\text {diag }}$ acts transversally to $K_{1}+K_{2}$ (condition (2.3)) guarantees that since $\Sigma$ is an integral of $K_{1}+K_{2}$, the composition $\mathbf{q}_{G_{\text {diag }}} \circ \Sigma$ is an immersion. Therefore $s=\mathbf{q}_{G_{\text {diag }}} \circ \Sigma$ is a 2-dimensional integral manifold of $I$.

We now show that $\gamma(a, b) \subset s((a, b) \times(a, b))$. All we need to do is set $t_{1}=t_{2}=t$ in equation (5.5). Since $\sigma(t)=\left(\sigma_{1}(t), \sigma_{2}(t)\right)$ we have

$$
\Sigma(t, t)=\mathbf{q}_{G_{\text {diag }}}\left(\sigma_{1}(t), \sigma_{2}(t)\right)=\mathbf{q}_{G_{\text {diag }}} \circ \sigma(t)=\gamma(t)
$$

and therefore $\gamma(a, b) \subset s((a, b) \times(a, b))$.

The proof of Theorem 1.1 is now simple. 
Proof. If $F\left(x, y, u, u_{x}, u_{y}, u_{x x}, u_{x y}, u_{y y}\right)=0$ is a hyperbolic PDE which is Darboux integrable after $k$ prolongations then $I^{\langle k\rangle}$, the $k^{\text {th }}$ prolongation of the rank 3 Pfaffian system $I$ in Theorem 3.1, is a hyperbolic Pfaffian system of class $3+k$ which is Darboux integrable [8]. Noncharacteristic initial data $\gamma:(a, b) \rightarrow M$ prolongs to non-characteristic initial data $\gamma^{\langle k\rangle}$ for $I^{\langle k\rangle}$. We then apply Theorem 5.3 to $I^{\langle k\rangle}$ to solve the initial value problem for $I^{\langle k\rangle}$ using the prolonged Cauchy data $\gamma^{\langle k\rangle}$. The solution $s$ to the Cauchy problem for the prolongation projects to the solution to the initial value problem for $I$ and hence $F$.

Finally, the solution given in Theorem 5.3 only requires computing the lift of the initial data $\gamma$ to an integral curve of $K_{1}+K_{2}$ on the product space $M_{1}+M_{2}$. This, by Theorem 2.1, only involves solving an equation of fundamental Lie type.

Remark 5.4. Given a Darboux integrable hyperbolic system $I$, Theorem 4.2 only guarantees that $I$ admits a local quotient representation in the sense that the action of $G$ is local and the quotient representation (4.2) only holds locally. In this case the implementation of Lemmas 5.1, 5.2 and Theorem 5.3 will only produce a local solution to the Cauchy problem. However it is still the case that if the Vessiot group $G$ is solvable, then this local solution can be found by quadratures.

At this point the reader may wish to refer to Appendix A to compare the results in this subsection with the classical approach to solving the Cauchy problem for Darboux integrable hyperbolic systems.

\subsection{Generalization to Darboux integrable systems}

In this section we outline how to solve the initial value problem in the general case of Darboux integrable Pfaffian systems which are not necessarily hyperbolic. A simple demonstration is given in Example 6.3.

A Pfaffian system $I$ is a called decomposable if about each point $p \in M$ there exists an open set $U \subset M$ and a coframe on $U$ given by

$$
\theta^{1}, \ldots, \theta^{r}, \widehat{\omega}^{1}, \ldots, \widehat{\omega}^{n_{1}}, \widehat{\tau}^{1}, \ldots, \widehat{\tau}^{p_{1}}, \breve{\omega}^{1}, \ldots, \breve{\omega}^{n_{2}}, \check{\tau}^{1}, \ldots, \breve{\tau}^{p_{2}}
$$

where $n_{1}+p_{1} \geqslant 2, n_{2}+p_{2} \geqslant 2, n_{1}, n_{2}, p_{1}, p_{2}, \geqslant 1$, with the properties (see Theorem 2.3 in [4]),

(i) the Pfaffian system is $I_{U}=\operatorname{span}\left\{\theta^{i}\right\}, 1 \leqslant i \leqslant r$;

(ii) the structure equations are

$$
\begin{aligned}
& d \theta^{i_{0}} \equiv 0 \quad \bmod I, \quad 1 \leqslant i_{0} \leqslant r_{1}, \\
& d \theta^{i_{1}} \equiv A_{a b}^{i_{1}} \widehat{\tau}^{a} \wedge \widehat{\omega}^{b} \quad \bmod I, \quad r_{1}+1 \leqslant i_{1} \leqslant r_{2}, \\
& d \theta^{i_{2}} \equiv B_{\alpha \beta}^{i_{2}} \check{\tau}^{\alpha} \wedge \check{\omega}^{\beta} \quad \bmod I, \quad r_{2}+1 \leqslant i_{2} \leqslant r ;
\end{aligned}
$$

(iii) and $I^{\prime}=\operatorname{span}\left\{\theta^{i_{0}}\right\}$.

Decomposable systems are generalizations of the hyperbolic systems defined in Section 3.

The Pfaffian systems $\widehat{V}, \breve{V}$ defined by

$$
\widehat{V}=\operatorname{span}\left\{\theta^{i}, \widehat{\tau}^{a}, \widehat{\omega}^{b}\right\} \quad \text { and } \quad \check{V}=\operatorname{span}\left\{\theta^{i}, \check{\tau}^{\alpha}, \check{\omega}^{\beta}\right\}
$$

are again called the characteristic or singular systems of a decomposable Pfaffian system $I$. As in the case of hyperbolic systems, a decomposable Pfaffian system $I$ is said to be Darboux integrable if $I^{\infty}=0$, and

$$
\widehat{V}+\check{V}^{\infty}=T^{*} M, \quad \hat{V}^{\infty}+\check{V}=T^{*} M .
$$

See [4] and Theorem 4.3 in [1]. 
Theorems 4.1 and 4.2 hold for Darboux integrable systems by simply dropping the codimension 2 requirement in Theorem 4.1, see [4] and [1]. This provides the canonical quotient representation of $I$ used in the analogue of Theorem 5.1.

The Cauchy initial value problem for a decomposable Pfaffian system consists of first prescribing an $\left(n_{1}+n_{2}-1\right)$-dimensional non-characteristic integral manifold $\gamma: S \rightarrow M$ which we will assume to be embedded. We then need to find an $\left(n_{1}+n_{2}\right)$-dimensional integral manifold $s: N \rightarrow M$ such that $\gamma(S) \subset s(N)$.

The full generalization of Theorem 1.1 is the following.

Theorem 5.5. Let I be a decomposable Darboux integrable Pfaffian system. The initial value problem for $I$ can be solved by integrating an equation of fundamental Lie type for the Vessiot group $G$. If $G$ is simply connected and solvable, then the initial value problem can be solved by quadratures.

The steps needed to prove Theorem 5.5 and solve the Cauchy problem are identical to those in Section 5.1. Lemmas 5.1, 5.2 and Theorem 5.3 have direct generalizations which appear in this section. However the proofs of these corresponding results are now significantly different. This is due to the fact that the condition that $S$ be non-characteristic for a general decomposable system is considerably more complex than condition (3.3) for a curve to be non-characteristic for a hyperbolic system.

Lemma 5.6. Let $I$ be a decomposable Darboux integrable Pfaffian system and (as in Theorem 4.2) let $\left(\left(K_{1}+K_{2}\right) / G_{\text {diag }},\left(M_{1} \times M_{2}\right) / G_{\text {diag }}\right)$ be the canonical quotient representation of $I$ where $G$ is the Vessiot group. Let $S \subset M$ be a non-characteristic integral manifold of $I$ of dimension $n_{1}+n_{2}-1$. Choose $x_{0} \in S$ and $\left(x_{1}, x_{2}\right) \in M_{1} \times M_{2}$ with $\mathbf{q}_{G_{\text {diag }}}\left(x_{1}, x_{2}\right)=x_{0}$, and let $L$ be the maximal integral manifold of the fundamental Lie system

$$
\left.\left(K_{1}+K_{2}\right)\right|_{\mathbf{q}_{G_{\text {diag }}}^{-1}(S)}
$$

through $\left(x_{1}, x_{2}\right)$. Then $L \subset M_{1} \times M_{2}$ is an $\left(n_{1}+n_{2}-1\right)$-dimensional non-characteristic integral manifold of $K_{1}+K_{2}$.

See [9] and [10] for more information on systems of equations of Lie type. The generalization of Lemma 5.2 is then the following.

Lemma 5.7. Let $L \subset M_{1} \times M_{2}$ be an $\left(n_{1}+n_{2}-1\right)$-dimensional embedded non-characteristic integral manifold of $K_{1}+K_{2}$, where $K_{1}$ and $K_{2}$ satisfy the conditions of Theorem 4.1. Then the manifolds $L_{i} \subset M_{i}$ defined by

$$
L_{i}=\pi_{i}(L)
$$

satisfy $\operatorname{dim} L_{1}=n_{2}$ and $\operatorname{dim} L_{2}=n_{1}$ and are integral manifolds of $K_{i}$.

Applying Lemmas 5.6 and 5.7 produces in a manner similar to Theorem 5.3 the following solution to the initial value problem.

Theorem 5.8. Let $S \subset M$ be a non-characteristic $\left(n_{1}+n_{2}-1\right)$-dimensional integral manifold for the Darboux integrable Pfaffian system I. Let $L \subset M_{1} \times M_{2}$ be a lift of $S$ to an integral manifold of $K_{1}+K_{2}$ satisfying the conditions in Lemma 5.6, and let $L_{i}=\pi_{i}(L)$. Then the smooth function $s: L_{1} \times L_{2} \rightarrow M$ defined by

$$
s\left(t_{1}, t_{2}\right)=\mathbf{q}_{G_{\text {diag }}}\left(t_{1}, t_{2}\right), \quad t_{1} \in L_{1}, \quad t_{2} \in L_{2},
$$

solves the local Cauchy problem for I about $x_{0}$ with Cauchy data $S \subset M$.

Lemma 5.6 and Theorem 5.8 establish Theorem 5.5. 
Remark 5.9. As this paper was near completion we obtained the preprint [16] which gives a detailed example of a Darboux integrable wave map system where the solution to the Cauchy problem reduces to the integration of a Lie system for $\mathrm{SL}(2, \mathbb{R})$. This is an excellent example of Theorem 5.8.

\section{Examples}

Example 6.1. For our first example we consider the Darboux integrable partial differential equation

$$
u_{x y}=\frac{u_{x} u_{y}}{u-x} .
$$

The closed-form general solution to this equation is well known (for example see [4]). Here however we will find the solution to (6.1) with initial Cauchy data by demonstrating the constructions in Theorems 4.2 and 5.3. Let $\gamma: \mathbb{R} \rightarrow M$ be the Cauchy data given by

$$
\begin{gathered}
\gamma(x)=\left(x=x, y=x, u=f(x), u_{x}=g(x), u_{y}=f^{\prime}(x)-g(x),\right. \\
\left.u_{x x}=g^{\prime}+\frac{g\left(f^{\prime}-g\right)}{x-f}, u_{y y}=f^{\prime \prime}-g^{\prime}+\frac{g\left(f^{\prime}-g\right)}{x-f}\right),
\end{gathered}
$$

where $f(x)$ has no fixed points.

The standard rank 3 Pfaffian system for the PDE in equation 6.1 is given on a 7 -manifold $M$ with coordinates $\left(x, y, u, u_{x}, u_{y}, u_{x x}, u_{y y}\right)$ by

$$
\begin{gathered}
I=\operatorname{span}\left\{\theta=d u-u_{x} d x-u_{y} d y, \theta_{x}=d u_{x}-u_{x x} d x-\frac{u_{x} u_{y}}{u-x} d y,\right. \\
\left.\theta_{y}=d u_{y}-\frac{u_{x} u_{y}}{u-x} d x-u_{y y} d y\right\} .
\end{gathered}
$$

With

$$
\widehat{\omega}=d x, \quad \widehat{\pi}=u_{x} d\left(\frac{u_{x x}}{u_{x}}+\frac{1}{u-x}\right), \quad \check{\omega}=d y, \quad \check{\pi}=u_{y} d\left(\frac{u_{y y}}{u_{y}}\right),
$$

we have

$$
d \theta \equiv 0, \quad d \theta_{x} \equiv \widehat{\omega} \wedge \widehat{\pi}, \quad d \theta_{y} \equiv \breve{\omega} \wedge \check{\pi} \bmod I,
$$

and

$$
\widehat{V}^{\infty}=\operatorname{span}\left\{d x, d\left(\frac{u_{x}}{u-x}\right), d\left(\frac{u_{x x}}{u_{x}}+\frac{1}{u-x}\right)\right\}, \quad \check{V}^{\infty}=\operatorname{span}\left\{d y, d\left(\frac{u_{y y}}{u_{y}}\right)\right\} .
$$

The canonical quotient representation for $I$ can be found be found using the algorithm in [4]. We find $M_{1}=\left\{\left(y, w, w_{y}, w_{y y}\right), w_{y}>0\right), M_{2}=\left\{\left(x, v, v_{x}, v_{x x}, v_{x x x}\right), v_{x}>0\right\}$, and

$$
\begin{aligned}
& K_{1}=\operatorname{span}\left\{d w-w_{y} d y, d w_{y}-w_{y y} d y\right\} \\
& K_{2}=\operatorname{span}\left\{d v-v_{x} d x, d v_{x}-v_{x x} d x, d v_{x x}-v_{x x x} d x\right\} .
\end{aligned}
$$

The Vessiot group $G$ is the 2-dimensional non-Abelian group $G=\left\{(a, b), a \in \mathbb{R}^{+}, b \in \mathbb{R}\right\}$ which acts on $M_{1}$ and $M_{2}$ by

$$
\begin{aligned}
& (a, b) \cdot\left(y, w, w_{y}, w_{y y}\right)=\left(y, a w-b, a w_{y}, a w_{y y}\right), \\
& (a, b) \cdot\left(x, v, v_{x}, v_{x x}, v_{x x x}\right)=\left(x, a v+b, a v_{x}, a v_{x x}, a v_{x x x}\right) .
\end{aligned}
$$


The quotient map $\mathbf{q}_{G_{\text {diag }}}: M_{1} \times M_{2} \rightarrow M$ can be written in coordinates as

$$
\begin{gathered}
\mathbf{q}\left(y, w, w_{y}, w_{y y} ; x, v, v_{x}, v_{x x}, v_{x x x}\right)=\left(x=x, y=y, u=x-\frac{v+w}{v_{x}},\right. \\
\left.u_{x}=\frac{(v+w) v_{x x}}{v_{x}^{2}}, u_{y}=-\frac{w_{y}}{v_{x}}, u_{x x}=D_{x}\left(u_{x}\right), u_{y y}=\frac{-w_{y y}}{v_{x}}\right) .
\end{gathered}
$$

A simple calculation shows that $\mathbf{q}_{G_{\text {diag }}}^{*}(I) \subset K_{1}+K_{2}$ and hence $I=\left(K_{1}+K_{2}\right) / G_{\text {diag. }}$. In this example this amounts to checking that

$$
u=x-\frac{v+w}{v_{x}}
$$

from equation (6.4) solves the PDE (6.1).

We proceed to solve the initial value problem using Theorem 5.3. First we must find the lift of the Cauchy data $\gamma$ given in (6.2) to an integral curve $\sigma$ of $K_{1}+K_{2}$. By using the second method of Remark 2.2. We find the 3-dimensional manifold $P=\mathbf{q}_{G_{\text {diag }}}^{-1}(\gamma(x)) \subset M_{1} \times M_{2}$ using (6.4) and (6.2) in terms of the parameters $x, v, v_{x}$,

$$
\begin{aligned}
P= & \left(y=x, w=(x-f(x)) v_{x}-v, w_{y}=\left(g(x)-f^{\prime}(x)\right) v_{x},\right. \\
& w_{y y}=\left(g^{\prime}(x)-f^{\prime \prime}(x)+\left(g(x)-f^{\prime}(x)\right) G(x)\right) v_{x} ; \\
& \left.x, v, v_{x}, v_{x x}=G(x) v_{x}, v_{x x x}=\left(G(x)^{2}+G^{\prime}(x)\right) v_{x}\right),
\end{aligned}
$$

where

$$
G(x)=\frac{g(x)}{x-f(x)}
$$

The restriction of the Pfaffian system from equation (6.3) to $P$ in (6.5) is then

$$
\left.\left(K_{1}+K_{2}\right)\right|_{P}=\operatorname{span}\left\{d v-v_{x} d x, d v_{x}-G(x) v_{x} d x\right\} .
$$

We choose the point (see Remark 2.2)

$$
\begin{aligned}
x_{0}=\left(x=0, y=0, u=f(0), u_{x}=g(0), u_{y}=f^{\prime}(0)-g(0),\right. \\
\left.u_{x x}=g^{\prime}(0)-\frac{g(0)\left(f^{\prime}(0)-g(0)\right)}{f(0)}, u_{y y}=f^{\prime \prime}(0)-g^{\prime}(0)-\frac{g(0)\left(f^{\prime}(0)-g(0)\right)}{f(0)}\right) \in S
\end{aligned}
$$

and $\left(x_{1}, x_{2}\right) \in M_{1} \times M_{2}$ to be (see (6.5))

$$
\begin{aligned}
\left(x_{1}, x_{2}\right)= & \left(y=0, w=-f(0), w_{y}=g(0)-f^{\prime}(0),\right. \\
& w_{y y}=g^{\prime}(0)-f^{\prime \prime}(0)+\left(f^{\prime}(0)-g(0)\right) G(0) ; \\
& \left.x=0, v=0, v_{x}=1, v_{x x}=G(0)\right) .
\end{aligned}
$$

These points satisfy $\mathbf{q}_{G_{\text {diag }}}\left(x_{1}, x_{2}\right)=x_{0}=\gamma(0)$. We now find the integral curve $\sigma$ of $\left.\left(K_{1}+K_{2}\right)\right|_{P}$ through $\left(x_{1}, x_{2}\right)$. This involves solving the Lie equation from (6.7) on $P$ subject to the initial data (6.8). With the $x$-coordinate as the parameter we find

$$
v_{x}=e^{\int_{0}^{x} G(t) d t}, \quad v=\int_{0}^{x} e^{\int_{0}^{s} G(t) d t} d s
$$

The explicit form for $\sigma: \mathbb{R} \rightarrow M_{1} \times M_{2}$ is found by inserting equation (6.9) in (6.5), giving

$$
\sigma(x)=\left(y=x, w=(x-f(x)) e^{\int_{0}^{x} G(t) d t}-\int_{0}^{x} e^{\int_{0}^{s} G(t) d t} d s, w_{y}=\left(g(x)-f^{\prime}(x)\right) e^{\int_{0}^{x} G(t) d t},\right.
$$




$$
\begin{aligned}
& w_{y y}=\left(g^{\prime}(x)-f^{\prime \prime}(x)+\left(g(x)-f^{\prime}(x)\right) G(x)\right) e^{\int_{0}^{x} G(t) d t} \\
& x=x, v=\int_{0}^{x} e^{\int_{0}^{s} G(t) d t} d s, v_{x}=e^{\int_{0}^{x} G(t) d t}, v_{x x}=G(x) e^{\int_{0}^{x} G(t) d t}, \\
& \left.v_{x x x}=\left(G(x)^{2}+G^{\prime}(x)\right) e^{\int_{0}^{x} G(t) d t}\right) .
\end{aligned}
$$

The curve $\sigma_{1}=\pi_{1} \circ \sigma$ is then easily determined from equation (6.10), which in terms of the parameter $y$ is

$$
\begin{aligned}
\sigma_{1}(y)=(y, w & =(y-f(y)) e^{\int_{0}^{y} G(t) d t}-\int_{0}^{y} e^{\int_{0}^{s} G(t) d t} d s, w_{y}=\left(g(y)-f^{\prime}(y)\right) e^{\int_{0}^{y} G(t) d t} \\
w_{y y} & \left.=\left(g^{\prime}(y)-f^{\prime \prime}(y)+\left(g(y)-f^{\prime}(y)\right) G(y)\right) e^{\int_{0}^{y} G(t) d t}\right) .
\end{aligned}
$$

The curve $\sigma$ in equation (6.10) also projects to the curve $\sigma_{2}=\pi_{2} \circ \sigma$

$$
\begin{gathered}
\sigma_{2}(x)=\left(x=x, v=\int_{0}^{x} e^{\int_{0}^{s} G(t) d t} d s, v_{x}=e^{\int_{0}^{x} G(t) d t}, v_{x x}=G(x) e^{\int_{0}^{x} G(t) d t},\right. \\
\left.v_{x x x}=\left(G^{\prime}(x)+G(x)^{2}\right) e^{\int_{0}^{x} G(t) d t}\right) .
\end{gathered}
$$

Now, according to equation (5.5) in Theorem 5.3, the solution to the PDE (6.1) with Cauchy data (6.2) is the image of the product of the curves in equation (6.11) and (6.12) in $M_{1} \times M_{2}$ under the map $\mathbf{q}_{G_{\text {diag }}}$ in equation (6.4). This gives

$$
\begin{aligned}
u & =x-\frac{v+w}{v_{x}}=x-\frac{\int_{0}^{x} e^{\int_{0}^{s} G(t) d t} d s-(f(y)-y) e^{\int_{0}^{y} G(t) d t}-\int_{0}^{y} e^{\int_{0}^{s} G(t) d t} d s}{e^{\int_{0}^{x} G(t) d t}} \\
& =x-\frac{\int_{y}^{x} e^{\int_{0}^{s} G(t) d t} d s-(f(y)-y) e^{\int_{0}^{y} G(t) d t}}{e^{\int_{0}^{x} G(t) d t}} \\
& =x+(f(y)-y) e^{\int_{x}^{y} G(t) d t}+e^{-\int_{0}^{x} G(t) d t}\left(\int_{x}^{y} e^{\int_{0}^{s} G(t) d t} d s\right),
\end{aligned}
$$

where $G(t)$ is given in equation (6.6). It is easy to check that this solves the Cauchy problem (6.2) for the PDE (6.1). One may interpret formula (6.13) as the analogue of d'Alembert's formula (in null coordinates) for equation (6.1).

Example 6.2. In this next example we write the standard rank 3 Pfaffian system $I$ for the non Monge-Ampere hyperbolic equation

$$
3 u_{x x} u_{y y}^{3}+1=0
$$

on a 7 -manifold $M$ with coordinates $\left(x, y, u, u_{x}, u_{y}, u_{x y}, u_{y y}\right)$ as

$$
I=\operatorname{span}\left\{d u-u_{x} d x-u_{y} d y, d u_{x}+\frac{1}{3 u_{y y}^{3}} d x-u_{x y} d y, d u_{y}-u_{x y} d x-u_{y y} d y\right\} .
$$

The construction of the canonical quotient representation for $I$ are given in [1] and we summarize the result here. On the 5 -manifolds $M_{1}$ with coordinates $\left(t, w, v, v_{t}, v_{t t}\right)$ and $M_{2}$ with coordinates $\left(s, q, p, p_{s}, p_{s s}\right)$ let

$$
\begin{aligned}
& K_{1}=\operatorname{span}\left\{d w-v_{t t}^{2} d t, d v-v_{t} d t, d v_{t}-v_{t t} d t\right\} \\
& K_{2}=\operatorname{span}\left\{d q-p_{s s}^{2} d s, d p-p_{s} d s, d p_{s}-p_{s s} d s\right\} .
\end{aligned}
$$


The action of the group $G=\mathbb{R}^{3}$ is given by

$$
\begin{aligned}
& (a, b, c) \cdot\left(t, w, v, v_{t}, v_{t t}\right)=\left(t, w+a, v+b+c t, v_{t}+c, v_{t t}\right), \\
& (a, b, c) \cdot\left(s, q, p, p_{s}, p_{s s}\right)=\left(s, q-a, p-b+c s, p_{s}+c, p_{s s}\right), \quad a, b, c \in \mathbb{R} .
\end{aligned}
$$

The quotient map $\mathbf{q}_{G_{\text {diag }}}: M_{1} \times M_{2} \rightarrow M$ is then given in these coordinates by

$$
\begin{aligned}
x= & -2 \frac{v_{t t}+p_{s s}}{s+t}, \quad y=\frac{1}{2}\left(v_{t t}-p_{s s}\right)(t+s)+p_{s}-v_{t}, \\
u= & -q-w+2 \frac{t v_{t}+s p_{s}-p-v}{s+t}\left(v_{t t}+p_{s s}\right) \\
& +\frac{1}{3}\left((2 s-t) v_{t t}^{2}+(2 t-s) p_{s s}^{2}-2(s+t) v_{t t} p_{s s}\right), \\
u_{x}= & p+v-t v_{t}-s p_{s}+\frac{s+t}{6}\left((2 t-s) v_{t t}+(2 s-t) p_{s s}\right), \\
u_{y}= & 2 \frac{s v_{t t}-t p_{s s}}{s+t}, \quad u_{x y}=\frac{1}{2}(t-s), \quad u_{y y}=\frac{2}{s+t} .
\end{aligned}
$$

It is straightforward matter to check $I=\left(K_{1}+K_{2}\right) / G_{\text {diag }}$.

Consider the Cauchy data $\gamma: \mathbb{R} \rightarrow M$ given by

$$
\gamma(\epsilon)=\left(x=0, y=\epsilon, u=f(\epsilon), u_{x}=g(\epsilon), u_{y}=f^{\prime}(\epsilon), u_{x y}=g^{\prime}(\epsilon), u_{y y}=f^{\prime \prime}(\epsilon)\right) .
$$

The four-dimensional manifold $P=\mathbf{q}_{G_{\text {diag }}}^{-1}(\gamma(\epsilon)) \subset M_{1} \times M_{2}$ can be computed from equation (6.15). Using the parameters $\epsilon, w, v, v_{t}$, we get

$$
\begin{aligned}
P=\left(t=h(\epsilon), w, v, v_{t}, v_{t t}=\frac{1}{2} f^{\prime} ; s=k(\epsilon), q=\frac{\left(f^{\prime}\right)^{2}}{2 f^{\prime \prime}}-w-f,\right. \\
\left.\quad p=g-\frac{f^{\prime}}{\left(f^{\prime \prime}\right)^{2}}+\epsilon k(\epsilon)+2 \frac{v_{t}}{f^{\prime \prime}}-v, p_{s}=v_{t}+\epsilon-\frac{f^{\prime}}{f^{\prime \prime}}, p_{s s}=-\frac{1}{2} f^{\prime}\right),
\end{aligned}
$$

where

$$
h(\epsilon)=\frac{1}{f^{\prime \prime}}+g^{\prime} \quad \text { and } \quad k(\epsilon)=\frac{1}{f^{\prime \prime}}-g^{\prime} .
$$

The restriction of $\left.\left(K_{1}+K_{2}\right)\right|_{P}$ is

$$
\left.\left(K_{1}+K_{2}\right)\right|_{P}=\operatorname{span}\left\{d w-\frac{\left(f^{\prime}\right)^{2}}{4} h^{\prime} d \epsilon, d v-v_{t} h^{\prime} d \epsilon, d v_{t}-\frac{f^{\prime}}{2} h^{\prime} d \epsilon\right\} .
$$

Taking the point $p_{0}=\left(\epsilon=0, v=0, w=0, v_{t}=0\right) \in P$ we find the maximal integral manifold $J$ of $\left.\left(K_{1}+K_{2}\right)\right|_{P}$ through $p_{0}$ to be

$$
v_{t}(\epsilon)=\frac{1}{2} \int_{0}^{\epsilon} h^{\prime} f^{\prime} d \xi, \quad v(\epsilon)=\int_{0}^{\epsilon}\left(\int_{0}^{\tau} h^{\prime} f^{\prime} d \tau\right) h^{\prime} d \xi, \quad w=\frac{1}{4} \int_{0}^{\epsilon}\left(f^{\prime}\right)^{2} h^{\prime} d \xi .
$$

Here $\mathbf{q}_{G_{\text {diag }}}\left(p_{0}\right)=\left(0,0, u=f(0), u_{x}=g(0), u_{y}=g^{\prime}(0), u_{y y}=f^{\prime \prime}(0)\right) \in S$. From Theorem 5.3, the solution to the initial value problem for (6.14) is then found using (6.16) and (6.15) to be

$$
\begin{aligned}
& x=\frac{f^{\prime}(\delta)-f^{\prime}(\epsilon)}{k(\delta)+h(\epsilon)}, \\
& y=\delta+\frac{1}{4} f^{\prime}(\epsilon)(h(\epsilon)+k(\delta))+\frac{1}{4} f^{\prime}(\delta)(h(\epsilon)-k(\delta))-\frac{1}{2} f^{\prime}(\delta) h(\delta)+\frac{1}{2} \int_{\epsilon}^{\delta} f^{\prime} h^{\prime} d \xi,
\end{aligned}
$$




$$
\begin{aligned}
u= & f(\delta)-\frac{f^{\prime}(\delta)^{2}}{2 f^{\prime \prime}(\delta)}+\frac{1}{4} \int_{\epsilon}^{\delta}\left(f^{\prime}\right)^{2} h^{\prime} d \xi+\frac{f^{\prime}(\epsilon)-f^{\prime}(\delta)}{h(\epsilon)+k(\delta)}\left(\frac{f^{\prime}(\delta) g^{\prime}(\delta)}{f^{\prime \prime}(\delta)}-g(\delta)-\frac{1}{2} \int_{\epsilon}^{\delta} f^{\prime} h^{\prime} h d \xi\right) \\
& +\frac{k(\delta)}{12}\left(2 f^{\prime}(\epsilon) f^{\prime}(\delta)+2 f^{\prime}(\epsilon)^{2}-f^{\prime}(\delta)^{2}\right)+\frac{h(\epsilon)}{12}\left(2 f^{\prime}(\delta)^{2}-f^{\prime}(\epsilon)^{2}+2 f^{\prime}(\epsilon) f^{\prime}(\delta)\right) .
\end{aligned}
$$

Example 6.3. In this last example we use the system of two partial differential equations

$$
u_{x z}=0, \quad u_{y z}=0
$$

to demonstrate the results in Section 5.2. The standard rank 4 Pfaffian system $I$ for this system on the 11-manifold $M$ with coordinates $\left(x, y, z, u, u_{x}, u_{y}, u_{z}, u_{x x}, u_{x y}, u_{y y}, u_{z z}\right)$ is given by

$$
\begin{gathered}
I=\operatorname{span}\left\{d u-u_{x} d x-u_{y} d y-u_{z} d z, d u_{x}-u_{x x} d x-u_{x y} d y,\right. \\
\left.d u_{y}-u_{x y} d x-u_{y y} d y, d u_{z}-u_{z z} d z\right\} .
\end{gathered}
$$

This is a decomposable Pfaffian system (Section 5.2) with $n_{1}=2, p_{1}=3$ and $n_{2}=1, p_{2}=1$. The canonical quotient representation for the Darboux integrable system $I$ is given by taking $M_{1}=J^{2}(\mathbb{R}, \mathbb{R})$ and $M_{2}=J^{2}\left(\mathbb{R}^{2}, \mathbb{R}\right)$ with

$$
\begin{aligned}
& K_{1}=\operatorname{span}\left\{d w-w_{z} d z, d w_{z}-w_{z z} d z\right\}, \\
& K_{2}=\operatorname{span}\left\{d v-v_{x} d x-v_{y} d y, d v_{x}-v_{x x} d x-v_{x y} d y, d v_{y}-v_{x y} d x-v_{y y} d y\right\} .
\end{aligned}
$$

Then $I=\left(K_{1}+K_{2}\right) / G$ where the action of $G=\mathbb{R}$ is given in coordinates by

$$
\begin{aligned}
& c \cdot\left(z, w, w_{z}, w_{z z}\right)=\left(z, w+c, w_{z}, w_{z z}\right), \\
& c \cdot\left(x, y, v, v_{x}, v_{y}, v_{x x}, v_{x y}, v_{y y}\right)=\left(x, y, v-c, v_{x}, v_{y}, v_{x x}, v_{x y}, v_{y y}\right), \quad c \in \mathbb{R} .
\end{aligned}
$$

The quotient map $\mathbf{q}_{G_{\text {diag }}}: M_{1} \times M_{2} \rightarrow M$ written in the above coordinates is easily found to be

$$
\begin{aligned}
& \mathbf{q}_{G_{\text {diag }}}\left(z, w, w_{z}, w_{z z} ; x, y, v, v_{x}, v_{y}, v_{x x}, v_{x y}, v_{y y}\right)=(x, y, z, u=w+v \\
& \left.\quad u_{x}=v_{x}, u_{y}=v_{y}, u_{z}=w_{z}, u_{x x}=v_{x x}, u_{x y}=v_{x y}, u_{y y}=v_{y y}, u_{z z}=w_{z z}\right) .
\end{aligned}
$$

Let $a(x, y)$ be a function of two variables and $k(\xi)$ a function of one, and let $S$ be the following non-characteristic two-dimensional integral manifold of $I$,

$$
\begin{aligned}
S= & \left(x, y, z=x+y, u=a(x, y), u_{x}=a_{x}-k(x+y), u_{y}=a_{y}-k(x+y),\right. \\
& u_{z}=k(x+y), u_{x x}=a_{x x}-k^{\prime}(x+y), x y=a_{x y}-k^{\prime}(x+y), \\
& \left.u_{y y}=a_{y y}-k^{\prime}(x+y), u_{z z}=k^{\prime}(x+y)\right) .
\end{aligned}
$$

We proceed using Lemma 5.6. The set $P=\mathbf{q}_{G_{\text {diag }}}^{-1}(S)$ is a 3-dimensional manifold. With parameters $x, y, v$ it is easily determined from equations (6.18) and (6.19) to be

$$
\begin{aligned}
P= & \left(z=x+y, w=a(x, y)-v, w_{z}=k(x+y), u_{z z}=k^{\prime}(x+y) ;\right. \\
& x, y, v, v_{x}=a_{x}-k(x+y), v_{y}=a_{y}-k(x+y), v_{x x}=a_{x x}-k^{\prime}(x+y), \\
& \left.v_{x y}=a_{x y}-k^{\prime}(x+y), v_{y y}=a_{y y}-k^{\prime}(x+y)\right) .
\end{aligned}
$$

On $P$ we have from $(6.17)$,

$$
\left.\left(K_{1}+K_{2}\right)\right|_{P}=\operatorname{span}\left\{d v-\left(a_{x}-k^{\prime}(x+y)\right) d x-\left(a_{y}-k^{\prime}(x+y)\right) d y\right\} .
$$

We now find, as in Lemma 5.6, the maximal 2-dimensional integral manifold $L$ for the Lie system in equation (6.21) subject to the initial conditions $(x=0, y=0, v(0,0)=a(0,0))$. We obtain

$$
v=a(x, y)-\int_{0}^{x+y} k(\xi) d \xi
$$


and this determines $L \subset M_{1} \times M_{2}$ in Lemma 5.6. With $w=a(x, y)-v$ from equation (6.20), where $v$ is given in (6.22), we find the manifolds $L_{i}$ in Lemma (5.7) to be (with $n_{1}=2$ and $n_{2}=1$ )

$$
\begin{aligned}
L_{1}=\pi_{1}(L)= & \left(z, w=\int_{0}^{z} k(\xi) d \xi, w_{z}=k(z), w_{z z}=k^{\prime}(z)\right), \\
L_{2}=\pi_{2}(L)= & \left(x, y, v=a(x, y)-\int_{0}^{x+y} k(\xi) d \xi, v_{x}=a_{x}-k(x+y), v_{y}=a_{y}-k(x+y),\right. \\
& \left.v_{x x}=a_{x x}-k^{\prime}(x+y), v_{x y}=a_{x y}-k^{\prime}(x+y), v_{y y}=a_{y y}-k^{\prime}(x+y)\right) . \quad(6.23)
\end{aligned}
$$

We now apply Theorem 5.8 to find the solution to the Cauchy problem. With $u=w+v$ from equation (6.18) and substituting for $w$ and $v$ in (6.23) we have

$$
u(x, y, z)=\int_{0}^{z} k(\xi) d \xi+a(x, y)-\int_{0}^{x+y} k(\xi) d \xi=a(x, y)+\int_{x+y}^{z} k(\xi) d \xi .
$$

\section{A The classical theory and a second approach}

In this appendix we recall the classical theory for solving the Cauchy problem for Darboux integrable hyperbolic systems as given in Remark 9.4 of [1] (demonstrated in [8]) and then relate this approach to the approach taken to solve the initial value problem given by Theorem 5.3. This will lead to an alternative but equivalent method of solution to the Cauchy problem which again requires solving equations of fundamental Lie type. Example A.3 below demonstrates the theory.

We denote by $\mathcal{I}$ the Pfaffian exterior differential system (EDS) generated by the sections of $I$. If $\mathbf{p}: M \rightarrow N$ is a surjective submersion we define the reduction of $\mathcal{I}$ by $\mathbf{p}$ as

$$
\mathcal{I} / \mathbf{p}=\left\{\theta \in \Omega^{*}(N) \mid \mathbf{p}^{*} \theta \in \mathcal{I}\right\} .
$$

If $G$ is a symmetry group then $\mathcal{I} / G=\mathcal{I} / \mathbf{q}_{G}$. For more details on EDS reduction see [3].

Let $\mathcal{I}$ be a Darboux integrable hyperbolic Pfaffian system and let $G, M_{i}, \mathcal{K}_{i}$ be the data for the canonical quotient representation of $\mathcal{I}$ as in Theorem 4.1 or 4.2. Let $\pi_{i}: M_{1} \times M_{2} \rightarrow M_{i}$ and let $\mathbf{q}_{G}^{i}: M_{i} \rightarrow M_{i} / G$ be the quotient maps. The compositions $\mathbf{q}_{G}^{1} \circ \pi_{1}$ and $\mathbf{q}_{G}^{2} \circ \pi_{2}$ are invariant with respect to the diagonal action of $G$ on $M_{1} \times M_{2}$ and therefore these maps factor through $\mathbf{q}_{G_{\text {diag }}}$. Accordingly we can define the surjective submersions $\mathbf{p}_{i}: M \rightarrow M_{i} / G$ so that the following diagram commutes (see also [1, equation (6.6), p. 21]):

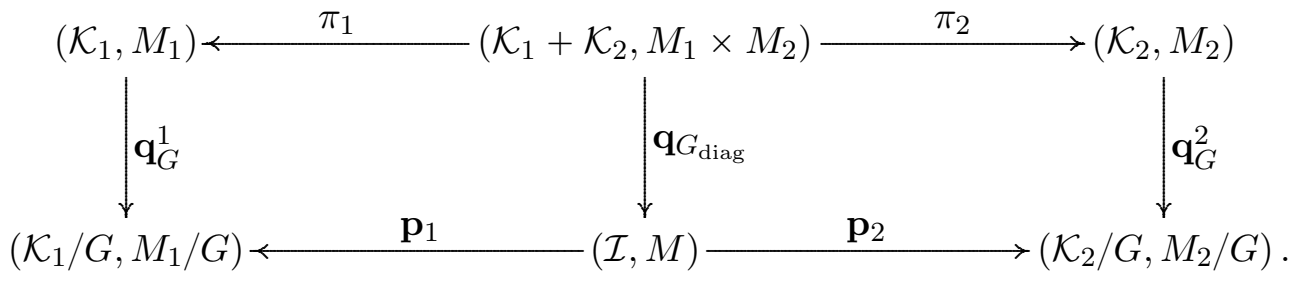

The commutativity of diagram (A.2) means that the Pfaffian systems $\mathcal{K}_{i}$ satisfy (see Theorem 3.1 in [1])

$$
\mathcal{K}_{i} / G=\mathcal{I} / \mathbf{p}_{i} \quad \text { and } \quad\left(\mathcal{K}_{1}+\mathcal{K}_{2}\right) / \pi_{i}=\mathcal{K}_{i} .
$$

It is important to note that the maps $\mathbf{q}_{G_{\text {diag }}}:\left(\mathcal{K}_{1}+\mathcal{K}_{2}, M_{1} \times M_{2}\right) \rightarrow(\mathcal{I}, M)$ and $\mathbf{q}_{G}^{i}:\left(\mathcal{K}_{i}, M_{i}\right) \rightarrow$ $\left(\mathcal{K}_{i} / G, M_{i} / G\right)$ in diagram (A.2) all satisfy the hypothesis of Theorem 2.1 except that $G$ may not act transversally to $K_{i}^{\prime}$. In this case $\mathcal{K}_{i} / G$ will not be a Pfaffian system. This is demonstrated in Example A.3. The maps $\mathbf{p}_{i}:(\mathcal{I}, M) \rightarrow\left(\mathcal{K}_{i} / G, M / G_{i}\right)$ are shown in [1] to be integrable extensions [7]. In particular the maps $\mathbf{p}_{i}$ map integral manifolds of $\mathcal{I}$ to integral manifolds of $\mathcal{K}_{i} / G$. 


\section{A.1 The classical method}

To describe the classical integration method we assume that the two integrable distributions $\breve{V}^{\infty}$ and $\widehat{V}^{\infty}$ are regular so that the two maps to the leaf spaces $\tilde{\mathbf{p}}_{1}: M \rightarrow M / \operatorname{ann}\left(\check{V}^{\infty}\right)$ and $\tilde{\mathbf{p}}_{2}: M \rightarrow M / \operatorname{ann}\left(\hat{V}^{\infty}\right)$ are smooth submersions. By using definition (A.1) we can then construct the following diagram

$$
\left(\mathcal{I} / \tilde{\mathbf{p}}_{1}, M / \operatorname{ann}\left(\check{V}^{\infty}\right)\right) \longleftrightarrow \tilde{\mathbf{p}}_{1}(\mathcal{I}, M) \underset{\tilde{\mathbf{p}}_{2}}{\longrightarrow}\left(\mathcal{I} / \tilde{\mathbf{p}}_{2}, M / \operatorname{ann}\left(\widehat{V}^{\infty}\right)\right) .
$$

The quotient spaces $M / \operatorname{ann}\left(\widehat{V}^{\infty}\right)$ and $M / \operatorname{ann}\left(\breve{V}^{\infty}\right)$ are thought of as the spaces of intermediate integrals in the sense that

$$
\breve{V}^{\infty}=\tilde{\mathbf{p}}_{1}^{*}\left(T^{*}\left(M / \operatorname{ann}\left(\check{V}^{\infty}\right)\right)\right) \quad \text { and } \quad \widehat{V}^{\infty}=\tilde{\mathbf{p}}_{2}^{*}\left(T^{*}\left(M / \operatorname{ann}\left(\widehat{V}^{\infty}\right)\right)\right) .
$$

The first of these equations implies that if $F: M / \operatorname{ann}\left(\check{V}^{\infty}\right) \rightarrow \mathbb{R}$, then $\tilde{\mathbf{p}}_{1}^{*}(d F)$ takes values in $\breve{V}^{\infty}$, and that a local basis of sections for $\breve{V}^{\infty}$ can be constructed in this way. A similar statement holds for $\widehat{V}^{\infty}$.

Let $\gamma:(a, b) \rightarrow M$ be a non-characteristic integral curve of the Darboux integrable hyperbolic system $\mathcal{I}$. We project the curve $\gamma$ into the spaces of intermediate integrals by

$$
\tilde{\gamma}_{i}=\tilde{\mathbf{p}}_{i} \circ \gamma
$$

The $\gamma_{i}$ are integral curves of $\mathcal{I} / \tilde{\mathbf{p}}_{i}$. Now let $N \subset M$ be the inverse image of the product curves

$$
N=\left(\tilde{\mathbf{p}}_{1}, \tilde{\mathbf{p}}_{2}\right)^{-1}\left(\tilde{\gamma}_{1}(a, b), \tilde{\gamma}_{2}(a, b)\right) .
$$

Then $\left.\mathcal{I}\right|_{N}$ is a Frobenius system. By determining the maximal integral manifold of $\left.\mathcal{I}\right|_{N}$ through a point $\gamma\left(t_{0}\right)$ of $\left.\mathcal{I}\right|_{N}$, one determines a local solution to the initial value problem. This is the classical method.

It is important to note that in the classical method the Vessiot group $G$ does not appear and there is no reason to expect that the Frobenius system $\left.\mathcal{I}\right|_{N}$ can be integrated by using equations of Lie type.

\section{A.2 A second method and the comparison}

The relationship between the classical method and Theorem 5.3 can be described using diagram (A.2) once we make one key observation. Namely that diagram (A.4) can be identified with the bottom row of diagram (A.2). To show how that identification can be made, suppose that the manifolds $M_{i}$ are connected, then the spaces of intermediate integrals $M / \operatorname{ann}\left(\tilde{V}^{\infty}\right)$ and $M / \operatorname{ann}\left(\hat{V}^{\infty}\right)$ may be identified with the two quotient spaces $M_{i} / G$. In particular it is shown in Theorem 6.1 of [1] that

$$
\breve{V}^{\infty}=\mathbf{p}_{1}^{*}\left(T^{*}\left(M_{1} / G\right)\right) \quad \text { and } \quad \hat{V}^{\infty}=\mathbf{p}_{2}^{*}\left(T^{*}\left(M_{2} / G\right)\right) .
$$

From diagram (A.2), part $(v)$ of Theorem 4.1, and (2.2) we have

$$
\operatorname{ker}\left(\mathbf{p}_{1 *}\right)=\mathbf{q}_{G_{\text {diag }} *}\left(0+T M_{2}\right)=\operatorname{ann}\left(\check{V}^{\infty}\right) .
$$

The fibres of $\mathbf{p}_{1}$ are of the form $\mathbf{q}_{G_{\text {diag }}}\left(x_{1}, M_{2}\right)$ which are connected since $M_{2}$ is connected. Therefore by Theorem 3.18 in [3],

$$
M / \operatorname{ann}\left(\check{V}^{\infty}\right) \cong M_{1} / G \quad \text { and } \quad \mathcal{I} / \tilde{\mathbf{p}}_{1} \cong \mathcal{K}_{1} / G
$$


where the first equivalence is by canonical diffeomorphism, and the second equivalence follows from the first equation in (A.3). Similarly we have

$$
M / \operatorname{ann}\left(\hat{V}^{\infty}\right) \cong M_{2} / G \quad \text { and } \quad \mathcal{I} / \tilde{\mathbf{p}}_{2} \cong \mathcal{K}_{2} / G
$$

With the identifications (A.7) and (A.8), we continue using diagram (A.2) to relate the classical solution to the Cauchy problem described above and Theorem 5.3. From the initial data $\gamma$ define the maps $\gamma_{i}:(a, b) \rightarrow M_{i} / G$ by

$$
\gamma_{i}=\mathbf{p}_{i} \circ \gamma
$$

Note that with the identifications in equation (A.7) and (A.8), the curves $\tilde{\gamma}_{i}$ in (A.5) and $\gamma_{i}$ in (A.9) are identified. The following lemma follows from the fact that the maps $\mathbf{p}_{i}:(\mathcal{I}, M) \rightarrow$ $\left(\mathcal{K}_{i} / G, M_{i}\right)$ are integrable extensions.

Lemma A.1. The curves $\gamma_{i}:(a, b) \rightarrow M_{i} / G$ are integral curves of $\mathcal{K}_{i} / G$.

Recall now that in Theorem 5.3 we choose a point $x_{0}=\gamma\left(t_{0}\right)$ and a point $\left(x_{1}, x_{2}\right) \in M_{1} \times M_{2}$ with $\mathbf{q}_{G_{\text {diag }}}\left(x_{1}, x_{2}\right)=x_{0}$. We then let $\sigma:(a, b) \rightarrow M_{1} \times M_{2}$ be the lift of $\gamma$ obtained by Theorem 2.1 through the point $\sigma\left(t_{0}\right)=\left(x_{1}, x_{2}\right)$. With

$$
\sigma_{i}=\pi_{i} \circ \sigma
$$

defined in equation (5.2) in Lemma 5.2, the solution to the initial value problem is given by $s\left(t_{1}, t_{2}\right)=\mathbf{q}_{G_{\text {diag }}}\left(\sigma_{1}\left(t_{1}\right), \sigma_{2}\left(t_{2}\right)\right)$ in equation (5.5).

Putting the curves $\gamma, \sigma, \sigma_{i}$ and $\gamma_{i}$ into the commutative diagram (A.2), we have

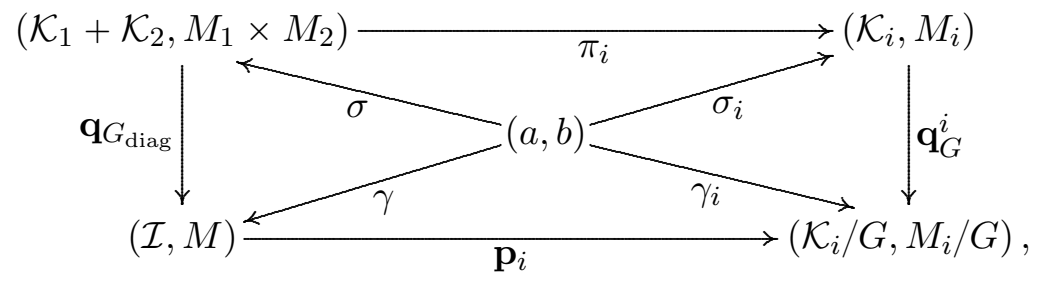

Since $\mathbf{q}_{G}^{i} \circ \sigma_{i}=\gamma_{i}$ and the initial points of $\sigma_{i}$ and $\gamma_{i}$ satisfy

$$
\sigma_{i}\left(t_{0}\right)=x_{i}, \quad \gamma_{i}\left(t_{0}\right)=\mathbf{p}_{i}\left(\mathbf{q}_{G_{\text {diag }}}\left(x_{1}, x_{2}\right)\right)=\mathbf{p}_{i}\left(x_{0}\right)=\mathbf{q}_{G}^{i}\left(\sigma_{i}\left(t_{0}\right)\right),
$$

we have by Theorem 2.1 and the commutative diagram (A.10) that $\sigma_{i}$ is the unique lift of $\gamma_{i}$ through $x_{i}$ which is an integral curve of $\mathcal{K}_{i}$. Consequently the curves $\sigma_{i}$ in Theorem 5.3 can be found from the curves $\gamma_{i}$, which are determined from the initial data $\gamma$ in (A.9), by solving two separate equations of fundamental Lie type. This gives us the following alternate method of solving the initial value problem.

Theorem A.2. Let $\gamma:(a, b) \rightarrow M$ be a non-characteristic integral curve for the Darboux integrable, hyperbolic Pfaffian system I with Vessiot group $G$. Let $\gamma_{i}:(a, b) \rightarrow M_{i} / G$ be the projection of $\gamma$ to the two spaces $M_{i} / G$, and let $\sigma_{i}:(a, b) \rightarrow M_{i}$ be the unique lift of $\gamma_{i}$ to an integral curve of $\mathcal{K}_{i}$ on $M_{i}$ satisfying the initial conditions in (A.11). Each curve $\sigma_{i}$ is found by solving an equation of fundamental Lie type and the function

$$
s\left(t_{1}, t_{2}\right)=\mathbf{q}_{G_{\text {diag }}}\left(\sigma_{1}\left(t_{1}\right), \sigma_{2}\left(t_{2}\right)\right), \quad t_{1}, t_{2} \in(a, b)
$$

solves the Cauchy problem for $\gamma$. 
At this point we can compare the approach to solving the Cauchy problem given in Theorem A.2 to the classical method in Section A.1. Starting with the Cauchy data $\gamma:(a, b) \rightarrow M$ to $\mathcal{I}$ we project $\gamma$ to curves $\tilde{\gamma}_{i}:(a, b) \rightarrow M_{i} / G$. The classical method then constructs the solution to the initial value problem by integrating the Frobenius system $\left.\mathcal{I}\right|_{N}$ where

$$
N=\left(\mathbf{p}_{1}, \mathbf{p}_{2}\right)^{-1}\left(\gamma_{1}(a, b), \gamma_{2}(a, b)\right) .
$$

On the other hand Theorem A.2 constructs the solution by using the quotient representation of $\mathcal{I}$ and lifting the projected curves $\gamma_{i}$ to integral curves of the EDS $\mathcal{K}_{i}$ on $M_{i}$. The lifting problem involves solving equations of fundamental Lie type for the Vessiot group $G$. It is at this point we see the fundamental difference between the classical method of solving the Cauchy problem and the one introduced here in Theorem A.2. This comparison shows how the Frobenius system in the classical solution can be integrated using equations of Lie type. As a consequence of our approach we can identify when the Cauchy problem for generic initial data can be solved by quadratures.

It is also worth noting that the projected curves $\gamma_{i}$ in equation (A.9) in the classical approach are thought of (through equation (A.6)) as prescribing the value of the intermediate integrals for $\mathcal{I}$ along the initial data. Integrating $\left.\mathcal{I}\right|_{N}$ in Section A.1 is then considered to be solving the "prescribed intermediate integral" problem. In Theorem A.2 we view the projected initial data $\gamma_{i}$ as prescribing "curvature invariants" of the curves $\sigma_{i}$ which we are trying to find. See Example A.3 below where this is demonstrated explicitly.

As our final remark we compare the two solutions to the Cauchy problem in Theorems 5.3 and A.2. In Theorem 5.3 we lift the Cauchy data $\gamma$ to $M_{1} \times M_{2}$ and then projects it to curves $\sigma_{i}:(a, b) \rightarrow M_{i}$. The solution $s$ is then constructed from these curves $\sigma_{i}$ in (5.5). In the approach Theorem A.2, we project the Cauchy data $\gamma$ to curves $\gamma_{i}:(a, b) \rightarrow M_{i} / G$ and then lift these curves to $\tilde{\sigma}_{i}:(a, b) \rightarrow M_{i}$. It is not difficult to show that the corresponding solutions $s$ in (A.12) and (5.5) are then identical.

Example A.3. In this example we write the standard rank 3 Pfaffian system $I$ for the Liouville equation

$$
u_{x y}=e^{u}
$$

on a 7 -manifold $M$ with coordinates $\left(x, y, u, u_{x}, u_{y}, u_{x y}, u_{y y}\right)$ as

$$
I=\operatorname{span}\left\{d u-u_{x} d x-u_{y} d y, d u_{x}-u_{x x} d x-e^{u} d y, d u_{y}-e^{u} d x-u_{y y} d y\right\}
$$

The intermediate integrals are given by

$$
\breve{V}^{\infty}=\operatorname{span}\left\{d y, d\left(u_{y y}-\frac{1}{2} u_{y}^{2}\right)\right\} \quad \text { and } \quad \widehat{V}^{\infty}=\operatorname{span}\left\{d x, d\left(u_{x x}-\frac{1}{2} u_{x}^{2}\right)\right\} .
$$

The details of the canonical quotient representation for $I$ are given in [1] and we summarize them here. Let $K_{1}$ and $K_{2}$ be the standard contact system on $J^{3}(\mathbb{R}, \mathbb{R})$ and $J^{3}(\mathbb{R}, \mathbb{R})$. In local coordinates $\left(y, w, w_{y}, w_{y y}, w_{y y y}\right)$ and $\left(x, v, v_{x}, v_{x x}, v_{x x x}\right)$ we have

$$
\begin{aligned}
& K_{1}=\operatorname{span}\left\{d w-w_{y} d y, d w_{y}-w_{y y} d y, d w_{y y}-w_{y y y} d y\right\} \quad \text { and } \\
& K_{2}=\operatorname{span}\left\{d v-v_{x} d x, d v_{x}-v_{x x} d x, d v_{x x}-v_{x x x} d x\right\} .
\end{aligned}
$$

Let

$$
\Gamma_{1}=\operatorname{span}\left\{\partial_{w}, \operatorname{pr}\left(w \partial_{w}\right), \operatorname{pr}\left(w^{2} \partial_{w}\right)\right\} \quad \text { and } \quad \Gamma_{2}=\operatorname{span}\left\{\partial_{v}, \operatorname{pr}\left(v \partial_{v}\right), \operatorname{pr}\left(v^{2} \partial_{v}\right)\right\}
$$


be the Lie algebras of vector-fields given by the prolongation of the standard infinitesimal action of $\mathfrak{s l}(2, \mathbb{R})$ acting on $w$ and $v$, and let

$$
\Gamma_{\text {diag }}=\left\{\partial_{w}-\partial_{v}, \operatorname{pr}\left(w \partial_{w}\right)+\operatorname{pr}\left(v \partial_{v}\right), \operatorname{pr}\left(w^{2} \partial_{w}\right)-\operatorname{pr}\left(v^{2} \partial_{v}\right)\right\}
$$

denote the diagonal action. On $M$, the open set where $v, v_{x}, w, w_{y}>0$, the distribution $\Gamma_{\text {diag }}$ is regular and $M / \Gamma_{\text {diag }}$ is 7 -dimensional. The quotient map $\mathbf{q}_{\Gamma_{\text {diag }}}$ can be written in coordinates as

$$
\begin{gathered}
\mathbf{q}_{\Gamma_{\text {diag }}}=\left(x=x, y=y, u=\log \frac{2 w_{y} v_{x}}{v+w}, u_{x}=\frac{v_{x x}}{v_{x}}-2 \frac{v_{x}}{v+w}\right. \\
u_{y}=\frac{w_{y y}}{w_{y}}-2 \frac{w_{y}}{v+w}, u_{x x}=\frac{v_{x x x}}{v_{x}}-\frac{v_{x x}^{2}}{v_{x}^{2}}-\frac{2 v_{x x}}{v+w}+\frac{2 v_{x}^{2}}{(v+w)^{2}}, \\
\left.u_{y y}=\frac{w_{y y y}}{w_{y}}-\frac{w_{y y}^{2}}{w_{y}^{2}}-\frac{2 w_{y y}}{v+w}+\frac{2 w_{y}^{2}}{(v+w)^{2}}\right) .
\end{gathered}
$$

We now construct the lower part of diagram (A.2) by computing the quotients $\left(\mathcal{K}_{i} / \Gamma_{i}, M_{i} / \Gamma_{i}\right)$. The projection maps into the differential invariants of $\Gamma_{i}$ on $M_{i}$ are

$$
\begin{aligned}
& \mathbf{q}_{\Gamma_{1}}\left(y, w, w_{y}, w_{y y}, w_{y y y}\right)=\left(y=y, \check{s}=\frac{w_{y y y}}{w_{y}}-\frac{3 w_{y y}^{2}}{2 w_{y}^{2}}\right) \quad \text { and } \\
& \mathbf{q}_{\Gamma_{2}}\left(x, v, v_{x}, v_{x x}, v_{x x x}\right)=\left(x=x, \widehat{s}=\frac{v_{x x x}}{v_{x}}-\frac{3 v_{x x}^{2}}{2 v_{x}^{2}}\right),
\end{aligned}
$$

where $\breve{s}$ and $\widehat{s}$ are the Schwarzian derivatives of $w(y)$ and $v(x)$. In terms of the differential invariants $\breve{s}$ and $\widehat{s}$, the algebraic generators of the differential systems $\mathcal{K}_{i}$ can be written

$$
\begin{aligned}
& \mathcal{K}_{1}=\left\langle d w-w_{y} d y, d w_{y}-w_{y y} d y, d w_{y y}-w_{y y y} d y, d \breve{s} \wedge d y\right\rangle_{\mathrm{alg}} \\
& \mathcal{K}_{2}=\left\langle d v-v_{x} d x, d v_{x}-v_{x x} d x, d v_{x x}-v_{x x x} d x, d \widehat{s} \wedge d x\right\rangle_{\mathrm{alg}} .
\end{aligned}
$$

The quotients are then quickly computed from (A.15) in the coordinates from (A.14) to be

$$
\mathcal{K}_{1} / \Gamma_{1}=\langle d \check{s} \wedge d y\rangle \quad \text { and } \quad \mathcal{K}_{2} / \Gamma_{2}=\langle d \widehat{s} \wedge d x\rangle .
$$

Equation (A.16) shows that an integral curve of $\mathcal{K}_{1} / \Gamma_{1}$ can be simply thought of as a choice of projective curvature $\breve{s}=G(y)$ and an integral curve of $\mathcal{K}_{2} / \Gamma_{2}$ is another choice of projective curvature $\widehat{s}=F(x)$.

The projection maps $\mathbf{p}_{i}: M \rightarrow M_{i} / \Gamma_{i}$ can be determined using the coordinates from equations (A.13) and (A.14). We find

$$
\breve{s}=\frac{w_{y y y}}{w_{y}}-\frac{3 w_{y y}^{2}}{2 w_{y}^{2}}=u_{y y}-\frac{1}{2} u_{y}^{2}, \quad \widehat{s}=\frac{v_{x x x}}{v_{x}}-\frac{3 v_{x x}^{2}}{2 v_{x}^{2}}=u_{x x}-\frac{1}{2} u_{x}^{2},
$$

and so

$$
\begin{aligned}
& \mathbf{p}_{1}\left(x, y, u, u_{x}, u_{y}, u_{x x}, u_{y y}\right)=\left(y=y, \breve{s}=u_{y y}-\frac{1}{2} u_{y}^{2}\right), \\
& \mathbf{p}_{2}\left(x, y, u, u_{x}, u_{y}, u_{x x}, u_{y y}\right)=\left(x=x, \widehat{s}=u_{x x}-\frac{1}{2} u_{x}^{2}\right) .
\end{aligned}
$$

Equations (A.17) and (A.18) shows that the (space of) intermediate integrals $\breve{V}^{\infty}$ and $\hat{V}^{\infty}$ are the pullback of the corresponding (space of) differential invariants of $\Gamma_{i}$ on $M_{i}$. This is the content of equation (A.6). 
We now consider the initial value problem for the non-characteristic integral manifold $\gamma$ : $\mathbb{R} \rightarrow M$ of $I$ given by

$$
\begin{gathered}
\gamma(x)=\left(x=x, y=x, u=f(x), u_{x}=g(x), u_{y}=f^{\prime}(x)+g(x),\right. \\
\left.u_{x x}=g^{\prime}(x)-e^{f(x)}, u_{y y}=f^{\prime \prime}(x)+g^{\prime}(x)-e^{f(x)}\right) .
\end{gathered}
$$

The projected curves $\gamma_{i}=\mathbf{p}_{i} \circ \gamma: M \rightarrow M_{i} / \Gamma_{i}$ are determined from (A.18) and (A.19) to be

$$
\gamma_{1}(y)=(y, \breve{s}=G(y)) \quad \text { and } \quad \gamma_{2}(x)=(x, \widehat{s}=F(x)),
$$

where

$$
\begin{aligned}
& G(y)=f^{\prime \prime}(y)+g^{\prime}(y)-e^{f(y)}-\frac{1}{2}\left(f^{\prime}(y)+g(y)\right)^{2} \quad \text { and } \\
& F(x)=g^{\prime}(x)-e^{f(x)}-\frac{1}{2} g(x)^{2} .
\end{aligned}
$$

The classical method of solving the initial value problem (A.19) given in Section A.1 is to find the integral manifolds of the system of $\mathcal{I}$ restricted to $\left(\mathbf{p}_{1}, \mathbf{p}_{2}\right)^{-1}(y, G(y), x, F(x))$. This restricted system is always completely integrable. By equations (A.9), (A.18) and (A.21) we have

$$
\left(\mathbf{p}_{1}, \mathbf{p}_{2}\right)^{-1}(y, G(y), x, F(x))=\left(x, y, u, u_{x}, u_{y}, u_{x x}-\frac{1}{2} u_{x}^{2}=F(x), u_{y y}-\frac{1}{2} u_{y}^{2}=G(y)\right),
$$

where $G(y)$ and $F(x)$ are given by (A.21). The restriction of $\mathcal{I}$ to this subset is the completely integrable Pfaffian system corresponding to the over-determined system of PDE

$$
\begin{aligned}
& u_{x x}-\frac{1}{2} u_{x}^{2}=F(x)=g^{\prime}(x)-e^{f(x)}-\frac{1}{2} g(x)^{2}, \quad u_{x y}=e^{u}, \\
& u_{y y}-\frac{1}{2} u_{y}^{2}=G(y)=f^{\prime \prime}(y)+g^{\prime}(y)-e^{f(y)}-\frac{1}{2}\left(f^{\prime}(y)+g(y)\right)^{2} .
\end{aligned}
$$

The solution to (A.22) solves the initial value problem (A.19) for the Darboux integrable equation $u_{x y}=e^{u}$.

The alternative method for solving the initial value problem (A.19) is presented in Theorem A.2. In this case we must construct integral curves $\sigma_{i}: \mathbb{R} \rightarrow M_{i}$ of the contact systems $\mathcal{K}_{i}$ such that $\mathbf{q}_{\Gamma_{i}} \circ \sigma_{i}=\gamma_{i}$, where $\gamma_{i}$ are given in (A.20). Using the expression for $\breve{s}$ and $\widehat{s}$ from equation (A.14) we seek curves $w(y)$ and $v(x)$ such that

$$
\begin{aligned}
& \frac{w_{y y y}}{w_{y}}-\frac{3 w_{y y}^{2}}{2 w_{y}^{2}}=G(y)=f^{\prime \prime}(y)+g^{\prime}(y)-e^{f(y)}-\frac{1}{2}\left(f^{\prime}(y)+g(y)\right)^{2}, \\
& \frac{v_{x x x}}{v_{x}}-\frac{3 v_{x x}^{2}}{2 v_{x}^{2}}=F(x)=g^{\prime}(x)-e^{f(x)}-\frac{1}{2} g(x)^{2} .
\end{aligned}
$$

A solution to (A.23) produces a solution to the initial value problem (A.19) by determining the curves $\sigma_{i}$ in the formula (A.12) in Theorem A.2.

It is worth pointing out that solving equations (A.23) is a classical reconstruction problem in projective differential geometry. That is, given the Schwarzian or projective curvature, $\widehat{s}=F(x)$, $x \in(c, d)$, construct a curve $\alpha:(c, d) \rightarrow \mathbb{R P}^{1}$ such that the Schwarzian derivative of $\alpha$ is $F(x)$. This is well known to be equivalent to solving an equation of fundamental Lie type for the simple Lie group $\operatorname{PSL}(2, \mathbb{R})$. 


\section{References}

[1] Anderson I.M., Fels M.E., Bäcklund transformations for Darboux integrable differential systems, arXiv:1108.5443.

[2] Anderson I.M., Fels M.E., Exterior differential systems with symmetry, Acta Appl. Math. 87 (2005), 3-31.

[3] Anderson I.M., Fels M.E., Symmetry reduction of exterior differential systems and Bäcklund transformations for PDE in the plane, Acta Appl. Math. 120 (2012), 29-60, arXiv:1110.5777.

[4] Anderson I.M., Fels M.E., Vassiliou P.J., Superposition formulas for exterior differential systems, Adv. Math. 221 (2009), 1910-1963.

[5] Bryant R.L., An introduction to Lie groups and symplectic geometry, in Geometry and Quantum Field Theory (Park City, UT, 1991), IAS/Park City Math. Ser., Vol. 1, Amer. Math. Soc., Providence, RI, 1995, $5-181$.

[6] Bryant R.L., Chern S.S., Gardner R.B., Goldschmidt H.L., Griffiths P.A., Exterior differential systems, Mathematical Sciences Research Institute Publications, Vol. 18, Springer-Verlag, New York, 1991.

[7] Bryant R.L., Griffiths P.A., Characteristic cohomology of differential systems. II. Conservation laws for a class of parabolic equations, Duke Math. J. 78 (1995), 531-676.

[8] Bryant R.L., Griffiths P.A., Hsu L., Hyperbolic exterior differential systems and their conservation laws. I, Selecta Math. (N.S.) 1 (1995), 21-112.

[9] Cariñena J.F., Grabowski J., Marmo G., Superposition rules, Lie theorem, and partial differential equations, Rep. Math. Phys. 60 (2007), 237-258, math-ph/0610013.

[10] Dubrov B.M., Komrakov B.P., The constructive equivalence problem in differential geometry, Sb. Math. 191 (2000), 655-681.

[11] Duzhin S.V., Lychagin V.V., Symmetries of distributions and quadrature of ordinary differential equations, Acta Appl. Math. 24 (1991), 29-57.

[12] Fels M.E., Exterior differential systems with symmetry, in Symmetries and Overdetermined Systems of Partial Differential Equations, IMA Vol. Math. Appl., Vol. 144, Springer, New York, 2008, 351-366.

[13] Gardner R.B., Kamran N., Characteristics and the geometry of hyperbolic equations in the plane, J. Differential Equations 104 (1993), 60-116.

[14] Kobayashi S., Nomizu K., Foundations of differential geometry, Vol. I, Wiley Classics Library, John Wiley \& Sons Inc., New York, 1996.

[15] Olver P.J., Applications of Lie groups to differential equations, Graduate Texts in Mathematics, Vol. 107, 2nd ed., Springer-Verlag, New York, 1993.

[16] Vassiliou P.J., Cauchy problem for a Darboux integrable wave map system and equations of Lie type, SIGMA, to appear. 